\title{
La universidad en un mundo con otros. De la extensión universitaria a las Prácticas Sociales Educativas
}

Victoria Noemí Kandel

\section{Resumen}

La fecha nos convoca. Cien años de una irrupción que sacudió a la universidad argentina y a buena parte de Latinoamérica. Cien años desde que la universidad fue interpelada, cuestionada, y en algunos casos reformulada. Un siglo en el cual la palabra "reforma universitaria" ha sido empleada de mil modos, y con sentidos y objetivos distintos. Una aparición en la historia, varios usos y una "tradición traicionada".

El movimiento reformista de Córdoba instaló una tradición, la tradición reformista, devenida en instituciones que regulan el sistema universitario argentino hasta hoy: la periodicidad de las cátedras, el cogobierno, la participación estudiantil, la asistencia libre, la docencia libre, la extensión universitaria.

Pero su rememoración evoca también una traición, un abandono, o tal vez un olvido voluntario, una forma selectiva y colectiva de decidir qué incluir en el proceso de la memoria y qué no. Pues el Movimiento Reformista, con su carácter antiimperialista y latinoamericanista ofreció una propuesta pedagógica y más particularmente una forma de pensar en la experiencia cotidiana del aula, una didáctica de la enseñanza universitaria.

Un planteo claro de aquellos años: la universidad no puede ser solo un centro de formación profesional porque eso sería reproducir un status quo, sino que debía ser-dicen sus textos y sus argumentos- un ámbito de formación de la persona y desde esta un motor de la transformación social. No puede separarse la Reforma Universitaria de una transformación social, cultural, política. La universidad tiene una responsabilidad en ese sentido, y el diálogo entre lo universitario y su contexto era concebido como imprescindible.

En estas páginas exploramos algunas de estas ideas sobre la universidad, la enseñanza y su articulación con las prácticas extensionistas, surgidas al calor de los debates de 1918 y nos preguntamos acerca de su vigencia y sus derivaciones en el contexto actual.

\section{Palabras clave:}

Movimiento Reformista de Córdoba, enseñanza, sociedad, extensión universitaria, prácticas sociales educativas. 
En la primera parte del texto, nos ocupamos de recuperar algunas ideas de los jóvenes reformistas sobre la enseñanza y el vínculo de la universidad con su entorno. Luego, en la segunda parte, -salteando un siglo- buscamos interpelar nuevamente al vínculo entre la universidad y su entorno, pero esta vez interrogando a las Prácticas sociales educativas.

\section{The university in a world with others. From College extension to the "Educational Social Practices"}

\section{Key words:}

Reformist Movement of Córdoba, education, society, university extension, social practices in education.

\begin{abstract}
The date calls us. It has been one hundred years ago since universities in Argentina and Latin America were irrupted by the "Movimiento Reformista de Córdoba". One hundred years since the university was questioned and, in some cases, reformulated. A century in which the word "university reform" has been used for different meanings and objectives. An irruption that founded a tradition... "a tradition betrayed".

The reformist movement of Córdoba installed a tradition, the reformist tradition, turned into institutions that regulate the Argentine university system until today: the periodicity of the chairs, co-government, student participation, free attendance, free teaching, and university extension.

But its remembrance also evokes a betrayal, a kind of forgetfulness, a selective and collective way of deciding what to include in the memory process and what not. For the Reformist Movement, with its anti-imperialist and Latin Americanist character, offered a pedagogical proposal and more particularly a way of thinking about the daily classroom experience, a didactic of higher education.
\end{abstract}

A clear statement of those years was: the university cannot be just a center of professional training because that would reproduce a status quo, but it should be -say their texts and their arguments- a field of formation of the person and from this an engine of social transformation. The university reform cannot be separated from a social, cultural or political transformation. The university has a responsibility in that sense, and the dialogue between the university and its context was conceived as essential.

In these pages we explore some of these ideas about vocational training and its articulation with extensions' practices that have been mentioned in 1918, and finally we ask ourselves about its validity and its derivations in the current context.

In the first part of the text, we explore some ideas of young reformers about teaching and the link of the university with its environment. Then, in the second part, -swinging a century- we seek to challenge again the link between the university and its environment, but this time interrogating Social educational practices.

\section{Parte 1}

\section{Los inicios de una tradición: la tradición reformista}

El Movimiento Reformista de Córdoba emerge, como sabemos, en un reducto conservador, tradicionalista y católico. Comenzó por un problema en apariencia menor, como 
fueron las exigencias de los estudiantes por una reforma en el sistema de provisión de las cátedras; y, por otro lado, una mayor vinculación entre la universidad y la realidad del país. Sin embargo, muy pronto los reformistas advirtieron que el reclamo por un cambio en la universidad debía ser más bien un reclamo por una renovación de las estructuras sociales y políticas del país. "No podrá separarse nunca la Reforma Universitaria de la Reforma Social, porque ambas fueron emprendidas simultáneamente y nacieron, por lo tanto, unidas", dice Julio V. González (citado por Portantiero, 1978, p.76).

Halperín Donghi afirma que,

\begin{abstract}
... la Reforma era, entre otras cosas, la protesta contra una universidad que se obstinaba -ahora más que antes-en permanecer al margen de la sustitución de grupos dirigentes culminante con los cambios políticos de 1916 ... Porque se daba en un contexto cultural nuevo, adquiría un sentido también renovado. (1962, p.127)
\end{abstract}

Para ello, la participación estudiantil resultaba fundamental, solo con esta presencia y este impulso "se daría solución de fondo y con perspectivas en el tiempo, al grave problema de la enseñanza superior" (Del Mazo, 1942, p. 32). El congreso de estudiantes, celebrado en julio de 1918, reclamaba autonomía universitaria frente al poder político en materia de decisiones académicas; elección de los cuerpos directivos y de las autoridades de la universidad por la propia comunidad con participación de los profesores, los graduados y los estudiantes en la composición de los órganos de gobierno; apertura de la universidad a los más amplios sectores de alumnos, facilitada por la asistencia libre y la asistencia social; modernización de la enseñanza, apertura de nuevas cátedras; concursos para proveer los cargos en las cátedras y periodicidad en las mismas.

La tarea del movimiento reformista se presenta como vasta y compleja, ya que apunta a modificar en sus bases a la universidad, y dar impulso, de este modo, a toda una serie de transformaciones que están pendientes en la sociedad, en el país y en América. La Reforma Universitaria no estaría completa si simultáneamente no se producían profundos cambios en la estructura social del país y del continente. Es por ello que unos años después, algunos autores se refirieron al fracaso de la Reforma Universitaria, en tanto faro que ilumina la lucha por el cambio social. "La Reforma afecta más a la forma que al fondo del problema educacional. Mientras subsista el actual régimen social, la Reforma no podrá tocar las raíces recónditas de tal problema" dice Del Mazo citando a Alejandro Korn (Del Mazo, 2000, p. 140). Y si los formatos de la enseñanza permanecen inmutables, petrificados... ¿estaremos ante una genuina reforma?

\title{
2. La democracia como premisa de la formación universitaria
}

El desafío de la formación profesional se resuelve, para los reformistas, apelando a la idea de democracia, en todos los aspectos y niveles que se pueda penetrar.

La idea de formación que subyace en el pensamiento reformista consiste en la internalización de la democracia en todos los órdenes del quehacer universitario. Sabemos que la democracia está ligada a la modernización de la universidad, ya que de ahí parte el reclamo estudiantil: "Nuestra democracia está constituida bajo la forma representativa y federal y todas las instituciones deben tender por lo tanto a adoptar igual forma política".

En este sentido se define también el Manifiesto Liminar:

La juventud ya no pide. Exige que se le reconozca el derecho a exteriorizar ese pensamiento propio en los cuerpos universitarios por medio de sus representantes. 
Está cansada de soportar a los tiranos. Si ha sido capaz de realizar una revolución en las conciencias, no puede desconocérsele la capacidad de intervenir en el gobierno de su propia casa. (Federación Universitaria de Córdoba [FUC], 1918)

La democracia es, pues, una experiencia universitaria, un aprendizaje constante, desde donde se despliega un doble propósito. De un lado, formar estudiantes que participen activamente del demos universitario, a través de los distintos canales institucionales que deberán estar previstos en un estatuto bajo la figura de la participación estudiantil en el gobierno universitario. Pero del otro lado, la formación democrática afecta y compromete al conjunto de la sociedad, ya que los estudiantes son, además, ciudadanos de una república. Precisamente por su juventud (y, en consecuencia, por su vigor y su ímpetu participativo), los estudiantes asumen en la república grande, plenamente sus funciones en tanto ciudadanos. Así, sostienen que:

La familia, la escuela, la universidad, deben ser, y esto es un postulado inatacable, escuelas de democracia, crisoles en que se fundan regios caracteres de ciudadanos libres, conscientes; yunque donde se doble la rebeldía insolente y al mismo tiempo, vivero donde surjan florecientes las revoluciones del pensamiento. (Del Mazo, 1942)

¿Cómo trasladar esta concepción de la democracia a la práctica cotidiana de la universidad? La asistencia libre ha sido una de las propuestas. En ella se condensan dos valores centrales que debe contener el ciudadano (claro está, ciudadano con doble pertenencia: a la república chica que es la universidad, y a la república grande: la libertad y la decisión).

Solo el amor puede dar la comprensión clara de las cosas, y tanto el amor a la ciencia como el amor a la casa de la ciencia, no nace de la autoridad de los reglamentos, sino que surge espontáneamente de la sustancia medular de cada vocación. Nunca puede tener la simpatía amplia del estudiante aquello que comienza por humillarlo, por someterlo a normas establecidas, encadenando, así, su libertad en lo que se refiere a la elaboración de sus conocimientos. Las modernas orientaciones pedagógicas nos llevan hacia la emancipación completa del educando, a la formación libre de la personalidad intelectual. Por otra parte, la asistencia voluntaria no solo destruye la mecanización de la enseñanza, sino que pone en manos del alumno un instrumento de defensa contra el profesor mediocre. (FUC, 1918)

Aparecen así calificativos como libre y maduro, para referir a los estudiantes universitarios. El estudiante es maduro, es un ciudadano pleno y, por lo tanto, debe ser libre en sus elecciones, sin ningún tipo de restricción. Las normas, las reglas que lo escolarizan, que lo ligan a las prácticas de la escuela, afectan a su libertad, ya que lo retrotraen al mundo de la infancia, de la incapacidad de decidir por sus propios medios. Los ecos de la Ilustración evocan a la figura de Kant y su definición acerca de "la mayoría de edad" como sinónimo de emancipación, autonomía y raciocinio. Recordemos que, a decir de Kant, el Iluminismo significaba el abandono de una situación nefasta ("la minoría de edad") y el ingreso a una nueva situación donde el hombre es capaz de pensar por sí mismo.

La asistencia libre enfatiza, pues, el derecho a elegir del que goza el estudiante. Y por intermedio de este mecanismo, el estudiante cuenta también con una herramienta que le permite juzgar el desempeño del docente. Esta herramienta supone la posibilidad de redistribuir el poder de juicio diseminándolo entre diferentes actores.

En este sentido, la universidad debe encarnar un aprendizaje ligado a la actitud del adulto, ciudadano libre y responsable, pero, además, trabajador. Para los reformistas, la asistencia libre se vincula también con la realidad adulta de los estudiantes en su carácter de trabajadores, ya que... 
La asistencia libre es, además, una disposición de orden social, pues es grande el número de los estudiantes que deben trabajar para sostener su vida. Correlacionada con la cátedra paralela, solo posible con la docencia libre, el alumno puede optar por enseñanzas a horario distinto y profesor diferente. El conjunto contribuye a la amplitud social de la universidad, a su democratización. (Del Mazo, 2000, p.65)

\section{El amor entre docente y alumno, y la idea de autoridad}

Muchos de los escritos de la Reforma comienzan con una denuncia para luego construir la propuesta. La relación del docente con los alumnos no escapa a esta generalidad.

Yo desde esta tribuna acuso públicamente a aquellos profesores empeñados en la tarea funesta de dictar sus cursos sin poseer la materia con ese amor a que he hecho referencia, y vuelvo a insistir y a repetir que nuestra eficiencia está en el amor con que hemos abordado estos problemas... y es una falta absoluta de vocación en el profesorado lo que introduce la anarquía en la enseñanza... nosotros somos estudiantes vocacionales y llegaremos, si es dado el caso algún día, a profesores por vocación... y la falta de vocación trae inmediatamente, la total desvinculación entre el profesor y los alumnos. (Hiram Pozzo citado por Del Mazo, 2000, p.41).

Desde esta perspectiva, la universidad se debe transformar en una experiencia en sí misma. Una experiencia que deja marcas, huellas, señales en quien la transita (Carli, 2006). Los estudiantes entendían que el encuentro con el docente puede convertirse en esta experiencia. Por ello el énfasis por diferenciar instrucción de enseñanza, profesor de maestro: "El día que los profesores enseñen más que su materia, amor a ella misma, diremos con orgullo que contamos con maestros". Sin embargo, admiten que esto no es lo que habitualmente ocurre, ya que mecanismos como la burocratización y la rutinización obstaculizan la posibilidad de que se generen genuinos encuentros entre docentes y alumnos. La falta de vocación es denunciada como uno de los principales obstáculos para la realización de una profunda experiencia formativa. Pero la vocación (o su carencia) afecta por supuesto a los profesores, pero también a los alumnos. Es importante destacar este elemento de autocrítica:

Pero también, para significar la sinceridad de nuestra tarea, digamos que no solamente el profesorado sin vocación es lo que promueve esta anarquía en los estudios de enseñanza superior, sino que a veces radica en los mismos alumnos la causa de esta anarquía y es, por aquellos alumnos que llegan a la universidad sin verdadera conciencia de sus funciones. Solo aspiran a un diploma que los habilite profesionalmente. (Hiram Pozzo citado por Del Mazo, 200o, p.41)

La noción de autoridad se configura desplazando la clásica idea del ejercicio de mando, el disciplinamiento y el temor, hacia el reconocimiento, la acogida, el cuidado y el amor. Es fácil advertir que cuando se busca autoridad no solo cuenta el temor, la sumisión o la violencia. A menudo cuando se pide autoridad se suele buscar guía, protección, cuidado, seguridad, control y orientación: Una definición de autoridad es precisamente la de alguien que utilizará su fuerza para cuidar a otros y atenderlos (Sennett, 1983). Estas definiciones sobre la autoridad desplazan la idea de fuerza por la idea de amor y de cuidado. El maestro es quien enseña, pero no quien enseña una determinada materia, sino quien enseña a amar esa materia, a amar la ciencia. El componente espiritual es fundamental para desarrollar plenamente la vocación del maestro, por eso afirman que:

La autoridad, en un hogar de estudiantes, no se ejercita mandando, sino sugiriendo y amando: enseñando. Si no existe una vinculación espiritual entre el que enseña y el que aprende, toda enseñanza es hostil y por consiguiente infecunda. Toda la educación es una larga obra de amor a los que aprenden. (FUC, 1918) 
La propuesta no se agota en la construcción de esa relación amorosa y cuidada entre el maestro y el alumno. Los reformistas apelan a mecanismos objetivos que permitirán el control y empoderan al estudiante ante el docente. De un lado, promovieron, como vimos, la docencia libre, y del otro, la periodicidad de la cátedra. Esto evitaría -según sus aseveraciones- que tanto los abusos como la mediocridad se perpetúen en el tiempo:

La inamovilidad del profesor en su sitial universitario conduce, muy a menudo, a la inmovilidad de sus ideas. Estando seguro de su puesto, con todas las ventajas que él proporciona, el deseo de perfeccionamiento disminuye y se apaga. El profesor perpetuo renueva muy difícilmente su caudal científico y se transforma, a la larga, en un ejemplar de museo. (Del Mazo, 200o, p. 64)

\section{4. "No es lo mismo saber que saber enseñar"}

Autoridad, amor, cuidado completan la figura del "verdadero maestro". En el Primer congreso nacional de estudiantes universitarios (celebrado en julio de 1918), se elaboró un documento denominado "Bases para la nueva organización de las universidades nacionales" (Del Mazo, 2000). Un capítulo se destina al estudio y posicionamiento de los estudiantes en relación con el sistema docente. Allí afirman de distintos modos lo que dice la frase que titula este apartado: no es lo mismo saber que saber enseñar.

En este sentido, reconocen dos condiciones esenciales para el ejercicio de la docencia: "la preparación científica -teórica y práctica- y la aptitud docente". La primera puede desarrollarse en soledad ("la soledad del gabinete") junto a libros, tubos de ensayo y lápices. Mientras que la segunda condición para el ejercicio de la docencia requiere siempre del encuentro con los otros, de un asomarse al mundo, mostrarse, pero también exponerse, exhibirse. Así, en el segundo caso, son los otros -los estudiantes- quienes juzgan el desempeño del docente a través de la concurrencia a clase: "los mejores docentes tendrán las aulas llenas, y los peores, verán los salones desiertos" (Del Mazo, 2000).

La vida en la universidad, y las experiencias que en la cotidianeidad impregnan el sentido de esa vida, son fundamentales para el logro de ese ansiado encuentro entre maestro y alumno. Es por ello fundamental el elemento temporal. Para los reformistas resultaba sugerente la idea de que el docente dedique gran parte de su tiempo a la enseñanza en la universidad. Estar dedicado enteramente a la universidad fue una de las propuestas que apuntaba a mejorar la calidad del vínculo docente-alumno:

En la intimidad educativa, por cultura de reunión se identifican los que aprendiendo enseñan y los que enseñando aprenden. No solo nadie hay que a la vez no puede ser en su medida alumno y maestro, sino que todo acto de comunión educativa, al conjugar autoridad y libertad, lleva en sí los posibles de un cultivo cada vez más extenso, penetrante y fecundo. Noble y activa concepción que a nadie excluye, que a todos incorpora, que a todos anima. (Del Mazo, 200o, p. 8)

La dedicación a la universidad implica un alto grado de compromiso, que los reformistas demandaban de los profesores, para poder - precisamente- profesar la docencia. Ahora bien, la profesión docente puede implicar también un retorno a una situación de aislamiento y enajenación respecto al mundo, que era precisamente aquello que se debía evitar, y se sumaría -además- un componente autoritario al imponer un tipo particular de vínculo con la universidad. Es por ello que una voz disonante se hizo oír en el Congreso de Estudiantes, precisamente para rebatir la idea de la profesión docente con dedicación completa: esta afirmación va en contra de los ideales mismos de la reforma ya que "significa regir la línea de conducta que todo hombre libre debe hacer a su arbitrio" (Del Mazo, 1942). Portantiero evoca una entrevista realizada a Deodoro Roca, en la cual él dijo: "El puro universitario es una cosa monstruosa". Claro 
que esta frase se enmarca en una reflexión sobre la "relación necesaria entre la rebelión estudiantil y el conjunto de las luchas populares" (Portantiero, 1978, p.76).

El saber y el saber enseñar se conjugan en el pensamiento reformista con un componente adicional vinculado con el saber práctico. El "saber hacer" que se adquiere necesariamente "haciendo", constituye uno de los elementos de mayor interés para el estudiante reformista. En esta preocupación se condensa la figura del graduado, quien representa "el adentro y el afuera de la universidad". Es por ello que el graduado compone el tercer elemento constitutivo del gobierno universitario, junto al docente y al alumno. Esta tríada conforma, pues, la ya mencionada experiencia universitaria al promover un conocimiento teórico complejo, pero a su vez una experiencia práctica motivada por la vida transcurrida fuera de la universidad.

La intervención de los graduados tendería a morigerar la desorientación o exceso de teoría en los planes de estudio y contribuiría a que se les diera a los estudiantes los conocimientos generales o prácticos que necesitan para la vida profesional.

\begin{abstract}
La dirección de la vida argentina está de hecho en manos de abogados, médicos, ingenieros y demás profesionales quienes, si bien tienen una preparación técnica que los capacita para el ejercicio de sus respectivas capacidades, viven en cambio en la más absoluta incomprensión de las estructuras históricas y sociales de la comunidad argentina, en el desconocimiento de aspectos fundamentales de su existencia y en una inestabilidad para apreciar el sentido y el ritmo de la nación (Baldrich, 1934, citado por Recalde, 2018, p.206)
\end{abstract}

La preocupación por la formación de la persona es recurrente. La formación técnica profesional necesita completarse con una formación del sujeto, con una conciencia respecto de la problemática social, de la condición latinoamericana, del pasado común y del futuro imaginado por los pueblos latinoamericanos. Por eso el Manifiesto liminar interpela “... A los hombres libres de Sudamérica”.

Nuevamente la tradición reformista. Tradición y traición. La tradición reformista reconoce a la extensión como una función principal entre las tres características del quehacer universitario: docencia, investigación y extensión. Extensión del conocimiento hacia quienes no pueden acceder a la universidad, extensión de las aulas y de la vida universitaria hacia los rincones en los cuales la luz universitaria no alcanza a iluminar. Lo social se cuela entre las funciones de la universidad. Surge así la función social de la universidad.

Ese es el origen. Origen revisado en la actualidad, al menos discursivamente. Si la extensión universitaria como práctica de interrelación entre el adentro y el afuera de lo universitario hoy recibe otras denominaciones (coproducción de conocimientos, intercambio, cooperación, vinculación o, también, compromiso social universitario), en las propuestas de 1918 tuvo como foco la promoción de un vínculo entre la universidad y los obreros, creando cursos sobre todas las cosas. Sostiene Del Mazo:

El movimiento de la Reforma no circunscribe los problemas universitarios a los limitados por los muros de la Universidad, sino que los considera como porción que integra la totalidad de los grandes problemas de cada una de nuestras naciones y de su conjunto. (2000, p.73)

Cuando los reformistas revisaron los acontecimientos, no dudaron en remarcar que se trataba de un proceso que perseguía un cambio político, social, cultural, más allá de sus raíces universitarias. Por lo tanto, podemos inferir que la preocupación por el cambio 
tiene que ver con una expectativa respecto de poder invertir un orden injusto y desigual, el latinoamericano, que ya por esos años constituía una preocupación recurrente.

Observemos esta bella frase de Deodoro Roca en 1936, reflexionando sobre el estudiante universitario de 1918:

La reforma fue todo lo que pudo ser. No pudo ser más de lo que fue, en dramas y actores. ¡Dio de sí todo! Dio pronto con sus límites infranqueables. Y realizó un magnífico descubrimiento. Esto solo la salvaría: al descubrir la raíz de su vaciedad y de su infecundidad notoria dio con este hallazgo: reforma universitaria es lo mismo que reforma social. (Portantiero, 1978, p. 77)

En ese sentido, la preocupación por entablar vínculos sólidos con el afuera, con lo nouniversitario, acompaña desde entonces, con apoyos y cuestionamientos, al quehacer universitario.

Comienza a aparecer la conciencia respecto de aquellos que necesitaban del saber universitario (de ahí la idea de extensión) y el compromiso de acercar el conocimiento hacia quienes lo requieren. Este vínculo con lo externo a lo universitario se mantuvo vivo, como decíamos, desde entonces, aunque asume matices y variaciones.

\section{Parte 2}

\section{La extensión como bisagra entre 1918 y nuestro tiempo actual}

Hasta aquí hemos evocado algunos pasajes del Movimiento Reformista de Córdoba de 1918. Convocamos fragmentos de sus consignas y de sus preocupaciones. Nos remontamos a su mirada sobre la enseñanza y el desafío de articular la formación disciplinar con la formación pedagógica. Al mismo tiempo, señalamos la importancia atribuida al vínculo con el mundo exterior: el mundo obrero, el mundo latinoamericano, el mundo social. El cambio universitario debía entrar en un diálogo con la realidad circundante (diálogo que -revisitado años después- según algunos de los protagonistas no sucedió).

En las siguientes páginas proponemos recuperar parte de ese diálogo entre "el adentro" y "el afuera" universitarios, visto desde la perspectiva de la extensión universitaria. Nos preguntamos cómo la universidad está tendiendo lazos con su entorno social, con su territorio. Y lo hacemos convocando a una figura recientemente creada en la Universidad de Buenos Aires (UBA): las Prácticas Sociales Educativas (PSE). Creemos que este instrumento recupera parte del espíritu con que en 1918 fue pensada la extensión universitaria, pero al mismo tiempo lo actualiza.

Las PSE surgieron en los últimos años como política académica impulsada por la UBA. El estudio de las PSE que aquí se presenta formó parte de una indagación sobre

1. El relevamiento se realizó entre los años 2012 y 2015 y fueron consultados directores de proyectos del Programa de Voluntariado Universitario y del Programa UBANEX. En ambos casos, se trata de proyectos de extensión universitaria, el primero con financiauniversitaria, el primero con financia-
miento del Ministerio de Educación de la Nación y el segundo con financiamiento propio. Han sido consultados directores de las facultades de Filosofía y Letras, Derecho, Ingeniería, Ciencias Exactas y Naturales, Agronomía y Ciencias Económicas. las transformaciones de las prácticas extensionistas a lo largo del último siglo en seis unidades académicas de la universidad mencionada. Para realizar esta indagación, se analizó la normativa y se realizaron entrevistas a directores de proyectos de extensión universitaria. $^{1}$

\section{El camino hacia la curricularización de la extensión universitaria}

El avance hacia la implementación de diversos mecanismos de institucionalización de prácticas de vinculación con el territorio (prácticas extensionistas) constituye una tendencia a nivel regional, como así también la configuración de instrumentos de 
política pública que apoyan, promueven y financian estas prácticas al interior de las universidades.

A modo de ejemplo, podemos mencionar el Programa de Voluntariado Universitario, creado en 2006 y actualmente renombrado como "Programa de Compromiso Social Universitario" (funciona bajo la órbita de la Dirección Nacional de Desarrollo Universitario y Voluntariado), fue creado con el propósito de promover el involucramiento de equipos de trabajo (compuestos por estudiantes y docentes de la universidad) con organizaciones comunitarias. La diferencia con las practicas extensionistas tradicionales es la articulación entre universidad y comunidad a través del trabajo con temáticas especificas contenidas en los programas de las asignaturas. De este modo, el propósito es vincular a la universidad con el territorio para fomentar intervenciones solidarias, pero mediadas por algún tipo de aprendizaje pertinente para cada una de las carreras que se dictan en la universidad.

En los últimos años algunas universidades argentinas han decido realizar reformas curriculares para incorporar prácticas solidarias educativas a los planes de estudios de todas sus carreras; entre ellas la UBA. ${ }^{2}$ Las Prácticas Sociales Educativas, fueron aprobadas por resolución 520 del Consejo Superior en 2010. Luego, mediante la Res. CS 172 de 2014 se estableció que la obligatoriedad debería comenzar en 2017, afectando a los estudiantes que ingresan en este año. ¿En qué consisten? Los estudiantes de la universidad deberán dedicar al menos cuarenta y dos horas a la realización de una práctica solidaria como requisito de egreso, práctica que deberá tener vínculo con algún contenido curricular perteneciente al plan de estudios de la carrera que se cursa.

No nos es posible avanzar en el análisis sobre los aspectos reglamentarios y la lógica de su implementación en cada unidad académica dado que aún se encuentra en instancia de deliberación con niveles diferentes de avance, pero conviene detenerse en dos elementos. Nos interesa aquí dar cuenta de los fundamentos de esta normativa, pues se propone disponer...

al servicio de la sociedad los saberes que produce y enseña, permitiendo fomentar la colaboración entre la universidad y la comunidad [...]

Además:

las Prácticas Sociales Educativas se constituyen en una herramienta eficaz para que los estudiantes desarrollen las habilidades y los valores de la ciudadanía, adquieran diversas visiones del mundo y alcancen una posición reflexiva frente a la realidad, a través de la participación, de nuevas y variadas formas, en la vida social. (Res. CS 172/14)

De acuerdo con la opinión de algunos directores de proyectos de voluntariado universitario o de extensión, lo atinado de las Prácticas Sociales Educativas se asocia al hecho de promover un vínculo -para algunos inexistente- entre la universidad y la sociedad. A continuación, se transcriben algunos testimonios que son elocuentes a la hora de valorar esta iniciativa: ${ }^{4}$

-“A mí me parece perfecto, yo creo que es una forma de llegar a la sociedad. Después cada cual tendrá su rumbo, pero haber pasado por esta instancia impacta en la formación de los alumnos. Independientemente de lo que hagan después con su profesión es importantísimo que esto lo tengan. Después también va a ser la posibilidad de que nos demos cuenta que la llegada a la sociedad no es algo que tiene que hacer el sociólogo o el psicólogo o el médico, sino que es algo que vos también podés hacerlo".
2. Otras que también lo han hecho son la Universidad Nacional del Litoral, Universidad Nacional de Río Cuarto, Universidad Nacional de La Pampa, Universidad Nacional de Mar del Plata, Universidad Nacional de Avellaneda, Universidad Nacional de Río Negro.
3. La reglamentación considera que dichas prácticas pueden insertarse en los siguientes modos: a. Como parte de los contenidos que se abordan en instancias de trabajos de campo o espacios similares, cuando se presenten fundamentos que sustenten su afinidad con el ejercicio de prácticas sociales; b. Como parte de un proyecto de extensión UBANEX pertinente; c. Como parte de otros programas y actividades desarrolladas por institutos o centros dependientes de las Unidades Académicas o el Rectorado; $d$. Como parte de las actividades desarrolladas a través de convenios con instituciones públicas y organizaciones de la sociedad civil; e. Como parte de propuestas que desarrollen diferentes equipos de cátedra, departamentos e institutos o; f. Como parte de proyectos de voluntariado universitario existente en organismos públicos. 4. Los testimonios que siguen a continuación son producto de entrevistas mantenidas con directores de proyec tos de extensión universitaria, de las Facultades de Agronomía, Veterinaria, Exactas, Sociales, Económicas y

Filosofía y Letras. Consultamos con ellos su opinión respecto de la aprobación de las prácticas sociales educativas por parte del C.S. de la UBA. 
Por otra parte, algunos entrevistados entienden que las PSE canalizan una necesidad latente de los estudiantes, vinculada con la idea de "devolución": la gratuidad interpela a algunos que se interrogan acerca de las posibilidades de devolver a la sociedad aquello que ella les brinda en materia de formación.

-“Me parece sano. Porque genera un vínculo distinto entre el profesional y la gente. Me parece que está bueno que el tipo no se quede encerrado en su unidad académica, me parece que está bien que el tipo tenga una conciencia de que existen reclamos sociales, de cómo se puede trabajar con la sociedad, con grupos sociales. En general los graduados de esta facultad tienen bastante conciencia de lo que es esta universidad, que es pública, que es gratuita, eso se da... y me parece que en general tienen ganas de retribuir. Muchos se quedan haciendo docencia justamente por eso, para retribuirle al Estado la formación que tuvieron. Y si encima se da posibilidad de retribuirle a la sociedad y no al Estado, me parece que es sano...".

Por último, algunos entrevistados entienden que las PSE permitirán jerarquizar y ofrecer reconocimiento institucional a una práctica que hasta el momento está bastante invisibilizada y no acreditada.

-“Yo tenía un poco de dudas con lo obligatorio, porque lo obligatorio a todos nos fastidia. Pero por la otra parte es la posibilidad de hacerlo conocido, visibilizarlo y darle un peso institucional que hasta ahora no tiene. Por ahí si pasan todos por las prácticas sociales, puede pasar que surjan cosas como esta: yo no hubiese participado, no me interesaba... y ahora que lo hice no me arrepiento. No hay que tenerle miedo en el sentido de la resistencia. Los alumnos no son tontos, yo creo que, si uno les ofrece algo de calidad, si ellos perciben que es bueno lo van a aprovechar".

-"Yo pienso que es bueno porque le va a dar la importancia que hoy no tiene a las actividades de extensión. Hoy mis amigos piensan que si voy al comedor es porque tengo tiempo para eso... creen que es una pérdida de tiempo, por eso me parece bien que la UBA le dé la importancia que hoy no tiene".

Sin embargo, algunos de nuestros entrevistados se han mostrado preocupados por la forma en que se implementará el proyecto al interior de las facultades. De hecho, es una de las dificultades que aún no se ha podido resolver de manera generalizada. Por un lado, resulta fundamental recuperar experiencias y recursos actualmente existentes, y por el otro, promover la creación de una "masa crítica" de docentes preparados para la realización de este tipo de proyectos en espacios donde hoy no los hay.

-“Nosotros desde acá, de económicas se generó la materia optativa de prácticas sociales solidarias como una suerte de prueba piloto, y también como una instancia de formación para poder hacer este salto a la obligatoriedad... formar un equipo docente, un equipo de coordinación que pueda trabajar con el desafío de poder involucrar en prácticas sociales a miles de alumnos, la facultad tiene cerca de sesenta mil estudiantes, entonces, no es sencillo coordinar un programa de prácticas sociales para todos. Entonces, desde 2010 estamos coordinando instancias de formación y de coordinación y de logística. Está funcionando bastante bien, hay cerca de doscientos alumnos por cuatrimestre y cerca de treinta jóvenes docentes y profesores a cargo... todavía hay gente que no está enterada, pero la materia se va enterando y saben de qué se trata. Pero sí hay cierta inquietud de qué es una práctica social.... Cada cuatrimestre se va anotando más".

Respecto a la implementación, las inquietudes de los entrevistados tienen que ver con el cuidado, tanto de los estudiantes como de la comunidad en su conjunto. 


\begin{abstract}
- “La reglamentación debería ser muy flexible para contener las formas de participación que ya existen y, por otro lado, contener a aquellos que van a desgano. Porque las consecuencias de trabajar a desgano pueden perjudicar mucho a las poblaciones más vulnerables...Creo que el proyecto debería cuidar mucho a la gente, además de a nuestros alumnos. ¿Qué pasa con aquella gente que va a hacer algo, pero mal... avalado por la UBA? ¡Ojo!”.
\end{abstract}

- “La idea me parece fantástica, el tema es cómo implementarla. Porque uno no puede andar largando gente a terreno, sin... hay una responsabilidad muy fuerte de la universidad de cuidar a los alumnos. Salir al campo requiere una formación previa muy fuerte. A nosotros no se nos ocurre salir al campo sin una preparación previa, hay que ser muy responsable en cómo se prepara a los alumnos. Nuestros alumnos en los primeros viajes van con algo de miedo. Otra cosa, el alumno se puede ver muy beneficiado con esta experiencia, de eso no me cabe duda. Pero es importante que las organizaciones también se vean beneficiadas de algún modo, ellas tienen que salir fortalecidas de ese vínculo. No se trata solo de mandar alumnos, sino de articular algo que sirva a todos".

\title{
A modo de conclusión
}

Los alcances del programa reformista han sido y continúan siendo hoy estudiados. Las universidades argentinas se han enmarcado en él, pero también lo han reinterpretado a lo largo de los últimos cien años.

En este texto propusimos espiar dicha tradición observando particularmente algunos de sus posicionamientos en torno a las formas y metas de la enseñanza y la extensión. Los textos de la Reforma enfatizaron su crítica hacia la enseñanza que se petrifica, a las clases magistrales, a la automatización, a la escasa formación pedagógica de los profesores. Al mismo tiempo, denuncian el aislamiento de lo universitario y establecen una agenda de vinculación con el entorno social apoyado en un programa antiimperialista y latinoamericano. La sensibilidad y la mirada hacia lo latinoamericano, lo local, lo que requiere de nuestra perspectiva universitaria, constituye el foco de sus reflexiones.

Buscamos mediante este texto enlazar la convocatoria reformista de 1918 que pensó a la universidad "desde adentro", tal como decía Pedro Krotsch (2001) con decisiones de política académica y curricular que actualmente están tomando algunas universidades argentinas, entre ellas, nuestra UBA. De este modo, entendemos que las actuales iniciativas que buscan curricularizar la extensión universitaria, y, por lo tanto, generar lazos formativos entre la universidad y sus entornos (en plural) recrea algunos elementos constitutivos del Movimiento Reformista de Córdoba. Tal vez logre profundizarlos, aún no podemos saberlo.

¿Cómo sostener un vínculo sólido, simétrico y perdurable entre la universidad y su entorno? Tal vez si se apuesta a centralizar la misión formativa propia de las universidades, haciendo dialogar a los saberes profesionales con los problemas reales, actuales, sentidos de una comunidad que podría beneficiarse con el saber universitario (Tapia, 2016). Asimismo, asumiendo que en la formación profesional hay ínsita una formación de una persona o un ciudadano que se solidariza y toma conciencia de la existencia de otro. El profesional/ciudadano/demos vuelve a escena, pero aún lo hace como desafío, como promesa. 


\section{Q Referencias bibliográficas}

»Carli, S. (2006). La experiencia universitaria y las narrativas estudiantiles. Una investigación sobre el tiempo presente. Revista Sociedad, (25). Buenos Aires, Argentina: Facultad de Ciencias Sociales y Prometeo.

»Del Mazo, G. (comp.). (1942). La Reforma Universitaria. La Plata, Argentina: Facultad de Ingeniería, UNLP.

»__. (2000). Estudiantes y Gobierno Universitario. San Luis, Argentina: Nueva editorial universitaria, UNSL.

» Federación Universitaria de Córdoba [FUC]. (1918). Manifiesto Liminar. Recuperado de :https://wold.fder.edu.uy/archivo/documentos/manifestoreforma-universitaria.pdf

» Halperín Donghi, T. (1962). Historia de la Universidad de Buenos Aires. Buenos Aires, Argentina: Eudeba.

"Krotsch, P. (2001). Educación Superior y Reformas Comparadas. Bernal, Argentina: Universidad Nacional de Quilmes.

» Portantiero, J. C. (1978). Estudiantes y política en América latina: el proceso de reforma universitaria, 1918-1938, Ciudad de México, México: Siglo Veintiuno.

» Recalde, A. (2018). La universidad y la integración sudamericana. En A. Roca y C. Schneider (comps.), El legado reformista en las nuevas universidades del conurbano. Inclusión, democracia, conocimiento. José C. Paz, Argentina: UNDAV y EDUNPAZ. Recupetrado de: https://www.unpaz.edu.ar/sites/default/files/CRES.pdf

"Sennett, R.ichard (1983). La autoridad. Madrid: Alianza.

» Tapia, N. (2016). Inserción curricular del Aprendizaje servicio en la educación superior. Clayss digital: Buenos Aires, Argentina. Recuperado de: http://www. clayss.org/o4_publicaciones/Insercion_curricular_EdSup.pdf

\section{Bibliografía de consulta}

"Kandel, V. (2017). Formar profesionales, formar ciudadanos. Reflexiones en torno a las prácticas sociales educativas. Revista de la Facultad de Ciencias Sociales de la UBA (94). Recuperado de: http://www.sociales.uba.ar/?page_id=47286

» Roca, A. y Schneider, C. (comps.). (2018). El legado reformista en las nuevas universidades del conurbano. Inclusión, democracia, conocimiento. Buenos Aires, Argentina: UNDAV. Recuperado de: https://www.unpaz.edu.ar/sites/default/files/CRES.pdf

\section{Victoria Noemí Kandel}

Doctora en Educación, Universidad de Buenos Aires. Magister en Ciencias Sociales con Orientación en Educación, Facultad Latinoamérica de Ciencias Sociales (FLACSO)Argentina; Licenciada en Ciencias de Políticas, Universidad de Buenos Aires. Profesora regular con dedicación exclusiva en la Universidad Nacional de Lanús y profesora en la Facultad de Derecho de la Universidad de Buenos Aires. Argentina. Correo electrónico: kandelv@gmail.com 